\title{
Simulação da difusão multicomponente durante a desidratação osmótica em maçã: determinação dos coeficientes de difusão pelo método simplex
}

\section{Simulation of the multicomponent diffusion during the osmotic dehydration of apple: determination of the diffusion coefficients by the simplex method}

\author{
Dionísio Borsato $^{1 *}$; Ivanira Moreira ${ }^{2}$; Rui Sérgio dos Santos Ferreira da Silva ${ }^{3}$; \\ Evandro Bona ${ }^{4}$; Marcelo Medre Nóbrega ${ }^{5}$; \\ Marcos Vinicios Roberto Pina ${ }^{6}$; Mariete Barbosa Moreira ${ }^{7}$
}

\begin{abstract}
Resumo
A perda de água e o ganho de açúcar foram modelados durante o processo de desidratação osmótica de cilindros de maçã. A transferência de soluto para a fruta e de água para a solução, foi modelada com base na $2^{\mathrm{a}}$ Lei generalizada de Fick para difusão simultânea e resolvida por meio do método de elementos finitos utilizando o software COMSOL Multiphysics 3.2. Os coeficientes de difusão principais e cruzados, a relação entre o coeficiente de transferência de massa e o de condutividade mássica bem como o número de Biot foram determinados na simulação com a aplicação do método de otimização simplex por meio da minimização dos desvios percentuais. $\mathrm{O}$ valor dos desvios entre os dados experimentais e simulados foi de $5,76 \%$ para a sacarose e $2,95 \%$ para a água. A simulação, desprezando o número de Biot, indicou que a resistência externa pode ser desconsiderada no fenômeno de transferência de massa estudado. O sistema desenvolvido para simular a difusão da água e do soluto permitirá o controle e a modulação do conteúdo de açúcar nos cilindros de maçã.

Palavras-chave: Difusão multicomponente, desidratação osmótica, método de elementos finitos
\end{abstract}

\section{Abstract}

Water loss and sugar gain were modeling during the osmotic dehydration process of cylinders of apple. The transfer of solute to the fruit and the water to the solution was based on Fick's 2nd law generalized for simultaneous diffusion and solved by the finite element method using the software COMSOL Multiphysics 3.2. The main and cross diffusion coefficients, the relationship between the mass transfer and mass conductivity coefficients, as well, the Biot number were determined on the simulation with the implementation of the simplex optimization method through minimization of the percentage deviations.

1 Docente, Dr. Departamento de Química, Universidade Estadual de Londrina. E-mail: dborsato@uel.br

2 Doutoranda, Programa de Pós-graduação do Departamento de Química, Universidade Estadual de Londrina. E-mail: ivanira@, uel.br

3 Docente, Dr. Departamento de Ciência e Tecnologia de Alimentos, Universidade Estadual de Londrina. E-mail ruisergio@uel. br

4 Universidade Tecnológica Federal do Paraná - Campus Campo Mourão. E-mail: ebona@utfpr.edu.br

5 Mestrando, Programa de Pós-graduação em Ciência de Alimentos, Universidade Estadual de Londrina. E-mail: marcelo.medre@ gmail.com

6 Bolsista de Iniciação científica, Universidade Estadual de Londrina. E-mail: marximpina@hotmail.com

7 Licenciada em Química, Universidade Estadual de Londrina. E-mail: marietequimica@yahoo.com.br

* Autor para correspondência 
The value of deviation between the experimental and the simulated data were 5,76\% for sucrose and 2,95 for water. The simulation despising the Biot number indicated that the resistance of the film can be disregarded in the phenomenon of mass transfer studied. The system developed to simulate solute and water diffusion will permit control and modulation of the sugar content in cylinder of apple.

Key words: Multicomponent diffusion. Osmotic dehydration. Finite element method.

\section{Introdução}

Uma das principais causas da deterioração de alimentos frescos e também conservados é a quantidade de água livre presente (MANNHEIM; LIU; GILBERT, 1994). A pesquisa e desenvolvimento dos métodos de preservação de alimentos concentram-se nos processos durante os quais a estrutura do produto não é alterada e o seu valor nutricional é mantido (FITO et al., 2001).

A desidratação osmótica, uma técnica bastante útil na conservação de frutas e vegetais, consiste em submeter o alimento sólido, inteiro ou em pedaços, a soluções aquosas (sais ou açúcares) de alta pressão osmótica para promover a remoção da água não ligada presente no alimento. No caso de frutas temse utilizado soluções de sacarose, com concentrações de 50 a $70^{\circ}$ Brix (TORREGGIANI, 1993). Neste processo há no mínimo dois fluxos simultâneos: a penetração dos sólidos da solução no material e o fluxo de água do material para a solução (YAO; LE MAGUER, 1997). Além do mais, a desidratação osmótica pode ser usada como um pré-tratamento antecedendo o congelamento, liofilização, secagem à vácuo e secagem com ar (PONTING, 1973; HAWKES; FLINK,1978; DIXON; JEN, 1977; NANJUNDASWAMY et al.,1978).

A desidratação osmótica caracteriza-se como um processo complexo de transferência de massa contra-corrente entre o tecido vegetal e a solução hipertônica. O fluxo da substância osmoativa penetrando no tecido modifica sua composição química. Há também um fluxo de substâncias nativas, no caso água, deixando o tecido (LEWICKI; LENART, 2007). A influência das principais variáveis do processo, como concentração e composição da solução osmótica, temperatura, tempo de imersão, procedimentos realizados como pré-tratamentos, agitação, natureza do alimento e sua geometria, relação solução e amostra no mecanismo de transferência de massa a na qualidade do produto tem sido estudados (AKTAS et al., 2006).

Este processo tem atraído atenção, devido as suas vantagens, incluindo: (1) retenção de cor e sabor, (2) manutenção na seletividade da parede celular, e (3) a necessidade de menos energia quando comparado com a secagem convectiva com ar quente. Apesar de todas essas vantagens, suas aplicações comerciais ainda são muito limitadas (LAZARIDES; KATSANIDES; NICOILAIDES, 1995; RAOULT-WACK, 1994).

Modelos de perda de água e ganho de soluto estão baseados na hipótese que a transferência de massa pode ser descrita pela equação de difusão de Fick ( $2^{\mathrm{a}}$ lei) em regime não estacionário. A equação de Fick estabelece uma relação entre o fluxo dos componentese os gradientes de concentração(GHEZ, 1988; RAOULT-WACK, 1994). Uma variedade de soluções para a 2a lei de Fick é apresentada de forma compreensiva por Crank (1975). Soluções análogas, para problemas de transferência de calor, que via de regra são rapidamente convertidas em soluções para a $2^{\mathrm{a}}$ lei de Fick podem ser encontradas no clássico de Carslaw e Jaeger (1959). Soluções particulares dependem de condições iniciais e das condições de contorno.

A transferência de solutos a partir de sólidos alimentares para um fluído adjacente ou para fluídos no interior do próprio sólido depende da difusão desses solutos através dos alimentos (SCHWARTZBERG; CHAO, 1982). Esta é a maior dificuldade para representar, por modelos simples, mudanças de concentração nos alimentos em processos de transferência de massa, devido à variedade de componentes individuais. 
Os coeficientes aparentes ou efetivos de difusão são determinados experimentalmente (ZORRILLA; RUBIOLO, 1994). A difusividade de solutos nos processos de extração ou infusão foi apresentada por Schwartzberg e Chao (1982).

Vários autores investigaram a transferência de calor e/ou massa em alimentos aplicando a formulação de elementos finitos (ZHANG et al., 1984; LOMAURO; BAKSHI, 1985; CHANDRA; SINGH, 1995). O método de elementos finitos (MEF), um conjunto de técnicas eficientes que obtém soluções numéricas de equações diferenciais, aparecem nos variados campos das ciências e em particular nos problemas de engenharia, física e química (CHUNG, 1978). O método é geral, no que diz respeito à geometria e às propriedades dos materiais. Corpos mais complexos e irregulares, compostos por diferentes materiais são facilmente representados, pois formas irregulares podem ser aproximadas em razão de cada elemento poder ser diferente (CHUNG, 1978).

A confiabilidade da solução obtida usando o MEF está diretamente relacionada com a discretização do modelo, que deve representar o problema físico considerado (LYRA, 1993).

A função de conveniência, uma técnica analítica para otimização de sistemas com várias respostas, é uma técnica fácil de ser aplicada, permitindo a avaliação subjetiva da importância das respostas (DERRINGER; SUICH, 1980). Métodos de busca direta, tais como otimização simplex, oferecem uma alternativa simples para otimização de respostas combinadas, necessitando, para isso, da definição do espaço experimental para a combinação dos fatores, além da função de compromisso de Derringer e Suich (1980).

O simplex é uma figura regular que se desloca sobre uma superfície de modo a evitar regiões de resposta não satisfatórias. No espaço n-dimensional o simplex representa um poliedro com faces planas contendo $n+1$ vértices, sendo n o número de variáveis independentes. O método como procedimento recorrente, tende a levar o simplex a um valor ótimo pela reflexão de pontos específicos. Nas vizinhanças do ótimo, o simplex pode apresentar contração com o objetivo de determinar uma posição mais precisa (BEVERIDGE; SCHECHTER, 1970). O método simplex é de fácil implantação nos processos automatizados. Sua aplicação é relativamente fácil, rápida, e permite com uma boa margem de segurança, localizar a região ótima, apesar de não oferecer informações claras com respeito ao comportamento das variáveis (EIRAS; ANDRADE, 1996).

O objetivo deste trabalho foi determinar os coeficientes de difusão e o número de biot, usando como modelo experimental a desidratação osmótica de maçãs.

\section{Material e Métodos}

Preparo das amostras

Foram utilizadas maçãs gala (Malus domestica Borkh) adquiridas em um supermercado local na região de Londrina - PR. Com o auxílio de um furador, foram retirados cilindros da maçã com 1,45 $\mathrm{cm}$ de diâmetro e estes cilindros foram cortados de modo a apresentar 2,5 cm de comprimento.

\section{Preparo da solução osmótica}

A solução osmótica foi preparada com uma concentração de açúcar de, aproximadamente, $60^{\circ}$ Brix. Foi utilizado como agente osmótico uma sacarose comercial da marca Alto Alegre refinado. Para garantir que a concentração da solução osmótica permanecesse constante e, também, a sua homogeneidade durante todo o processo de desidratação o volume de solução utilizado foi 20 vezes maior do que o volume ocupado pelos cilindros de maçã (KHIN; ZHOW; YEO, 2007; DEROSSI et al., 2008). Antes do início e no término do processo de desidratação foi retirada uma amostra da solução para quantificação do açúcar presente. 
Como a solução de sacarose apresentou densidade maior que os cilindros de maçã, foi utilizado um suporte reticulado de polietileno para garantir que as amostras permanecessem imersas durante todo o processo de desidratação permitindo, com isso, manter as mesmas condições de contorno em todas as faces. O estudo da desidratação osmótica foi feito em solução estática, em recipiente fechado para evitar a evaporação, mantida sob refrigeração para a manutenção da temperatura de $20 \pm 1^{\circ} \mathrm{C}$ durante todo o processo.

\section{Amostragem}

As amostras cilíndricas de maçã foram coletadas em intervalos compreendidos nas 33 horas de experimento.

\section{Determinação da umidade}

A porcentagem de umidade das amostras de maçã foi determinada por secagem das amostras em estufa a $100^{\circ} \mathrm{C}$ até peso constante (RICHARDSON, 1985; AOAC, 1984).

\section{Determinação da sacarose}

A sacarose foi determinada em um refratômetro de ABBE (PANADES et al., 2008).

Formulação em elementos finitos aplicada ao processo de desidratação osmótica

Para a formulação da difusão simultânea em elementos finitos foram feitas as seguintes considerações ou hipóteses simplificadoras:

Modelou-se a difusão de um soluto em um cilindro de maçã tridimensional que ocupa o volume $\Omega \subset \mathrm{R}^{3}$, associado a um sistema de coordenadas cartesianas $\mathrm{x}, \mathrm{y}, \mathrm{z}$, com origem no centro geométrico da peça de maçã.
O coeficiente de difusão ou difusividade mássica foi considerado constante em relação à concentração (independente do tempo e posição).

Nessas condições, propõe-se analisar o processo de desidratação de maçã, considerando-a imersa numa solução aquosa estática contendo sacarose na proporção em massa de $60 \%$. A concentração $\mathrm{C}_{1}(\mathrm{x}, \mathrm{y}, \mathrm{z}, \mathrm{t})$ do soluto utilizado e a concentração $\mathrm{C}_{2}(\mathrm{x}, \mathrm{y}, \mathrm{z}, \mathrm{t})$ de água num ponto $\mathrm{P}(\mathrm{x}, \mathrm{y}, \mathrm{z}) \in \Omega$ e num instante $t$, podem ser descritas a partir das equações de Onsager (1945) para as concentrações dos solutos:

$$
\begin{aligned}
& \frac{\partial C_{1}}{\partial t}=D_{1} \nabla^{2} C_{1}+D_{2} \nabla^{2} C_{2} \\
& \frac{\partial C_{2}}{\partial t}=D_{2} \nabla^{2} C_{1}+D_{2} \nabla^{2} C_{2}
\end{aligned}
$$

onde $\mathrm{D}_{\mathrm{ii}}$, são os coeficientes principais, $\mathrm{D}_{\mathrm{ij}}$ são os coeficientes cruzados, que acoplam os fluxos e $\nabla^{2}()=.\nabla . \nabla($.$) é o operador laplaciano.$

No processo de desidratação, as condições iniciais são dadas por:

$$
\begin{aligned}
& C_{1}(x, y, z, 0)=C_{1,0} \\
& C_{2}(x, y, z, 0)=C_{2,0} \quad x, y, z \in \Omega
\end{aligned}
$$

onde $\mathrm{C}_{1,0}$ e $\mathrm{C}_{2,0}$ são conhecidas. As condições de contorno para o processo de difusão sem agitação ficam sendo (LUNA; BRESSAN, 1986):

$$
\begin{gathered}
\frac{\partial C_{1}( \pm R, t)}{\partial \eta}=\frac{h_{m}}{\lambda_{m}}\left[C_{1}-C_{1, s}\right] \\
\frac{\partial C_{2}( \pm R, t)}{\partial \eta}=\frac{h_{m}}{\lambda_{m}}\left[C_{2}-C_{2, s}\right] \\
\text { com x, y, z } \in \partial \Omega, \mathrm{t}>0
\end{gathered}
$$

onde, $\mathrm{h}_{\mathrm{m}}\left(\mathrm{g} / \mathrm{cm}^{2} . \mathrm{h}\right)$ é o coeficiente de transferência de massa, $\lambda_{\mathrm{m}}(\mathrm{g} / \mathrm{cm} . \mathrm{h})$ a condutividade mássica; $\partial \Omega$ o conjunto de pontos da superfície que contorna o 
cilindro de maçã; $\mathrm{C}_{1, \mathrm{~s}}$ e $\mathrm{C}_{2, \mathrm{~s}}$, as concentrações dos solutos que banham o cilindro de maçã e $\partial / \partial \eta$ o operador da derivada normal. Os coeficientes $h_{m}$ e $\lambda_{\mathrm{m}}$ estão relacionados com o número de Biot pela equação

$$
B=\frac{h_{m} \cdot R}{\lambda_{m}} ; \quad i=1,2,3
$$

O processo de desidratação foi simulado utilizando uma malha contendo elementos tetraédricos e assumiu-se como constante o volume da amostra em todo o processo.

\section{Teste estatístico}

A comparação entre dados teóricos e experimentais do teor de sólidos e de água foi feita por meio do desvio percentual (BONA et al., 2007), como forma de avaliar a qualidade do ajustamento.

$$
\% \text { desvio }=100 \sqrt{\sum_{i=1}^{N}\left[\left(\frac{\bar{C}_{\text {calc }}-\bar{C}_{\text {exp }}}{\bar{C}_{\text {exp }}}\right)_{i}\right]^{2} \frac{1}{N}}
$$

onde: $\bar{C}_{\text {calc }}=$ concentração média estimada pela solução numérica;

$\bar{C}_{\text {exp }}=$ concentração experimental média;

$\mathrm{N}=$ número de observações consideradas.

\section{Método de otimização simplex}

O simplex no espaço $n$-dimensional é um sólido com faces planas contendo $(n+1)$ vértices, onde $n$ é o número de variáveis independentes. O método é um procedimento recorrente, que tende a levar o simplex a um valor ótimo com a reflexão de pontos específicos. Uma vez nas vizinhanças do ótimo, o simplex pode apresentar contração com objetivo de determinar uma posição mais precisa (BEVERIDGE; SCHECHTER, 1970).

Ajuste dos coeficientes de difusão e do número de Biot

Os coeficientes de difusão e o número de Biot foram ajustados através do método de otimização simplex (WALTERS et al., 1999) associado às funções de desejabilidade (DERRINGER; SUICH, 1980). Um algoritmo de otimização (BONA et al., 2000) propôs combinações para os coeficientes de difusão e número de Biot. Estes valores foram avaliados através do MEF. As concentrações de sacarose e água simuladas foram então comparadas aos resultados experimentais mediante teste de avaliação estatística (equação 4). Os desvios percentuais encontrados foram reavaliados pelo método de otimização que forneceu novas combinações de valores com o objetivo de minimizar o desvio entre os valores estimados e os experimentais. O procedimento foi repetido até a estabilização dos valores calculados para os desvios, coeficientes de difusão e número de Biot. A Tabela 1 a seguir mostra os limites das variáveis independentes utilizados na otimização simplex que foram obtidos a partir de ensaios preliminares e consulta na literatura especializada (DIXON; JEN, 1977; KHIN et al., 2007).

Tabela 1. Limite inferior e superior das variáveis independentes

\begin{tabular}{lcc}
\hline Variável independente & Limite inferior & Limite superior \\
\hline $\mathrm{D}_{11} \times 10^{-10}\left(\mathrm{~m}^{2} / \mathrm{s}\right)$ & 1,10 & 2,00 \\
$\mathrm{D}_{22} \times 10^{-10}\left(\mathrm{~m}^{2} / \mathrm{s}\right)$ & 1,10 & 2,00 \\
$\mathrm{D}_{12} \times 10^{-11}\left(\mathrm{~m}^{2} / \mathrm{s}\right)$ & 1,15 & 10 \\
$\mathrm{D}_{21} \times 10^{-11}\left(\mathrm{~m}^{2} / \mathrm{s}\right)$ & 1,15 & 10 \\
Número de Biot (adimensional) & 10 & 100 \\
\hline
\end{tabular}




\section{Programação computacional}

Para simulação do processo de desidratação osmótica foi utilizado o software COMSOL Multiphysics, e foi processado em um computador Core 2 Duo com 2 Ghz de memória.

\section{Resultados e Discussão}

A desidratação osmótica na maçã foi realizada utilizando uma solução estática. A solução foi mantida sob refrigeração para controle e manutenção da temperatura $\left(20 \pm 1^{\circ} \mathrm{C}\right)$ durante todo o processo. A Tabela 2 mostra as características iniciais da solução osmótica utilizada.

A Tabela 3 mostra os valores correspondentes ao teor de umidade, de sólidos totais e do volume médio dos cilindros de maçã antes de iniciar o processo experimental de desidratação osmótica.

A malha tetraédrica utilizada na simulação foi gerada automaticamente, sendo composta por 4585 elementos com 14224 graus de liberdade, como é mostrado na Figura 1.

Tabela 2. Características iniciais da solução osmótica*

\begin{tabular}{ll}
\hline Temperatura durante a desidratação & $20 \pm 1^{\circ} \mathrm{C}$ \\
Densidade $\left(\mathrm{g} / \mathrm{cm}^{3}\right)$ & 1,2875 \\
Sacarose $\left(\mathrm{g} / 100 \mathrm{~g}_{\text {sacarose +água }}\right)$ & 58,5 \\
Água $\left(\mathrm{g} / 100 \mathrm{~g}_{\text {sacarose +água }}\right.$ & 41,5 \\
Volume de maçã : volume de solução & $1: 20$ \\
\hline
\end{tabular}

* as características iniciais e finais da solução são as mesmas.

Tabela 3. Características relevantes da amostra antes de iniciar a desidratação osmótica

\begin{tabular}{lc}
\hline Umidade $(\mathrm{g} / 100 \mathrm{~g}$ de amostra $)$ & $86,50 \%( \pm 0,01)$ \\
Teor de sólidos $(\mathrm{g} / 100 \mathrm{~g}$ de solução) & $13,50 \%( \pm 0,01)$ \\
Volume médio da amostra $\left(\mathrm{cm}^{3}\right)$ & $4,126( \pm 0,1)$ \\
\hline
\end{tabular}

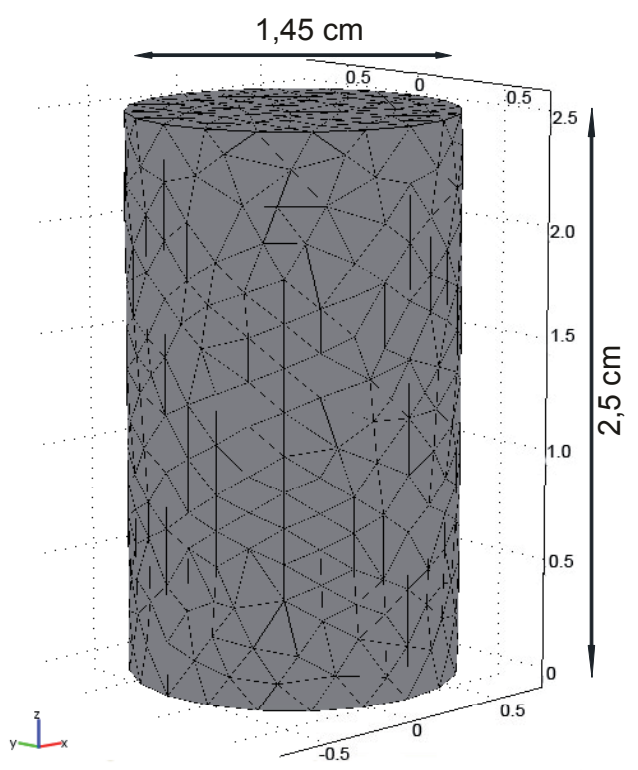

Figura 1. Dimensão média do cilindro de maçã submetido à solução osmótica, malha tetraédrica e convenção adotada para os eixos imaginários para identificação da simetria. 
Com a malha fornecida pelo programa computacional e o esquema de discretizaçãotemporal utilizado foi obtida a estimativa dos coeficientes de difusão e número de Biot pelo método de otimização simplex (BONA et al., 2000). O método simplex inicializou a busca com 6 conjuntos de valores isto é $\mathrm{n}+1$ utilizando as restrições mostradas na tabela 1. Cada conjunto foi utilizado na simulação pelo programa COMSOL Multiphisics e os valores obtidos foram comparados com os valores experimentais por meio do desvio percentual (equação 4). Os dois valores dos desvios (respostas) foram codificados de zero a um e transformados por meio de uma função de desejabilidade representada por uma média geométrica dos dois valores obtidos (CASTRO et al., 2003). O conjunto que apresentou o maior desvio calculado foi substituído por um novo conjunto de valores gerados pelo simplex e, o sistema recorrente, através de reflexões e/ou contrações levou o sistema ao valor ótimo (BONA et al., 2000). A estabilização dos valores dos desvios, em relação aos dados experimentais e simulados, pode ser observado na Figura 2.

A Figura 2 mostra que os desvios foram estabilizados, após 20 interações para a sacarose e 15 para a água.

A estabilização dos valores dos coeficientes de difusão e do número de Biot, após 30 interações, pode ser observada na Figura 3.

As estimativas dos valores dos coeficientes de difusão, da relação entre os coeficientes de filme e da condutividade mássica bem como o número de Biot, otimizadas pelo método simplex, encontramse na Tabela 4.
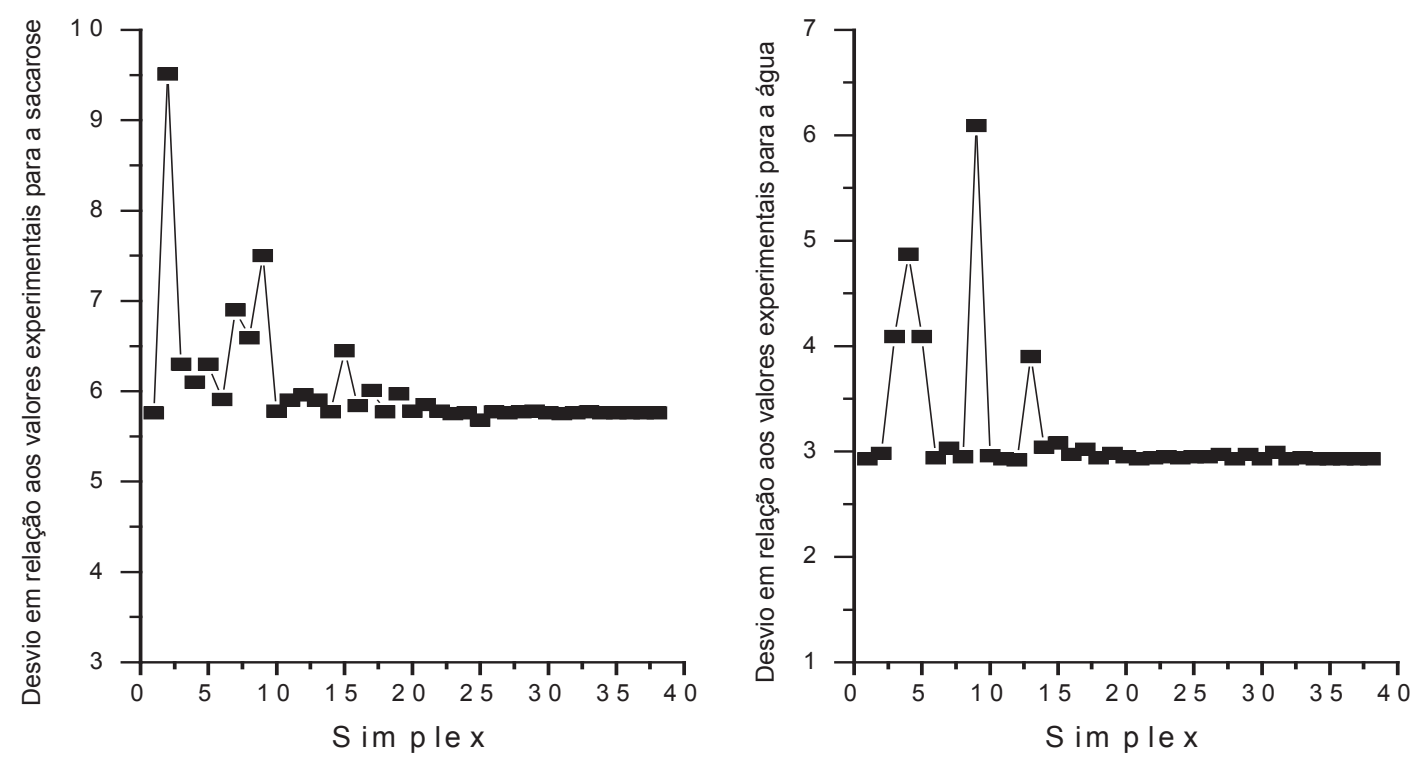

Figura 2. Estabilização dos desvios durante a aplicação do método simplex. 

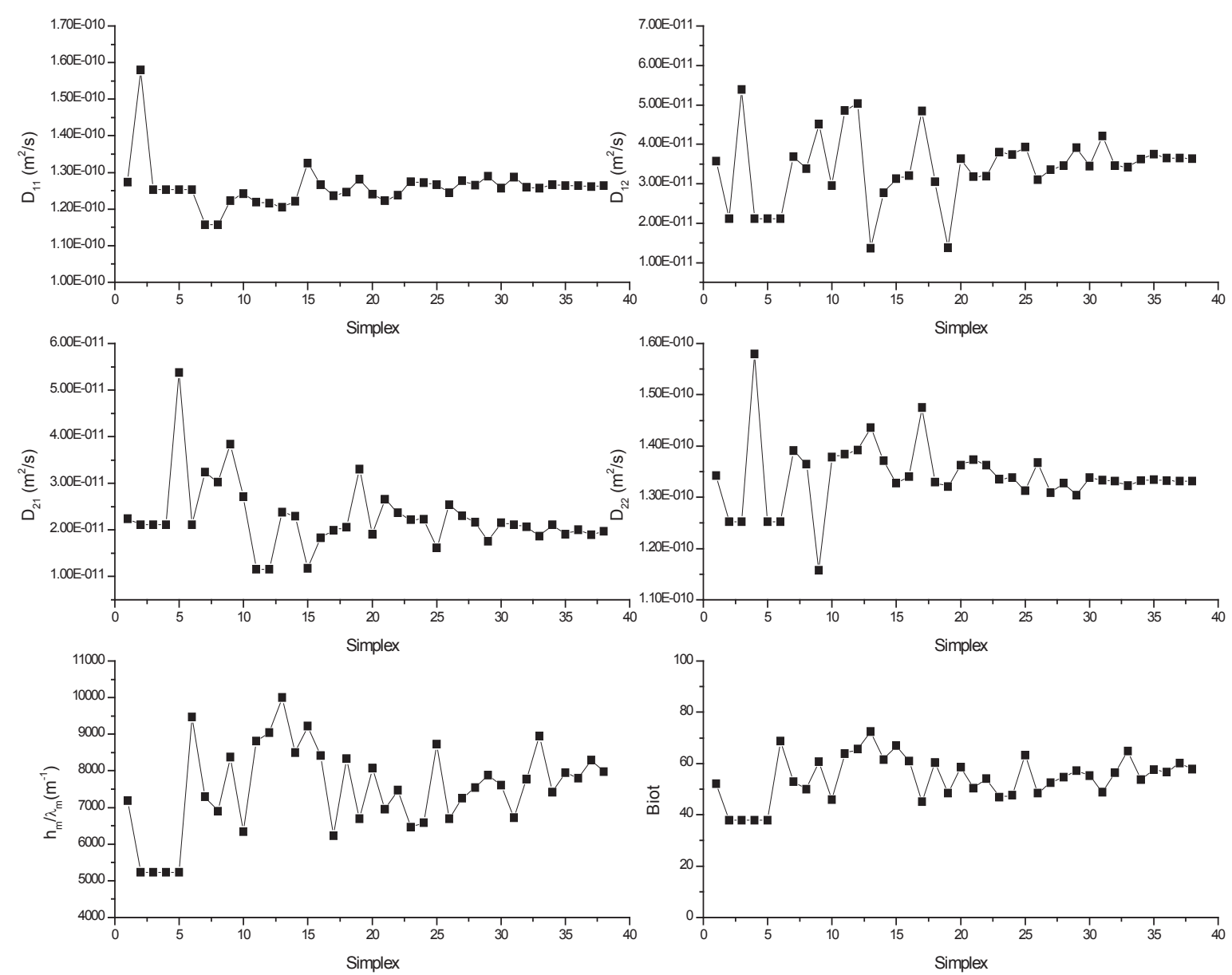

Figura 3. Estabilização dos parâmetros estimados através do método simplex.

Tabela 4. Coeficientes de difusão ajustados para o processo de desidratação osmótica da maçã.

Coeficientes principais $\left(\mathrm{m}^{2} / \mathrm{s}\right)$

Coeficientes cruzados $\left(\mathrm{m}^{2} / \mathrm{s}\right)$

Desvio (\%)

$\mathrm{h}_{\mathrm{m}} / \lambda_{\mathrm{m}}\left(\mathrm{m}^{-1}\right)$

Biot

\begin{tabular}{cc} 
Sacarose & Água \\
\hline $1,2662 \times 10^{-10}\left(\mathrm{D}_{11}\right)$ & $1,3125 \times 10^{-10}\left(\mathrm{D}_{22}\right)$ \\
$3,92361 \times 10^{-11}\left(\mathrm{D}_{12}\right)$ & $1,62037 \times 10^{-11}\left(\mathrm{D}_{21}\right)$ \\
5,76 & 2,95
\end{tabular}

8729,11

63,28605 
Os coeficientes cruzados apresentam uma ordem de grandeza inferior aos coeficientes principais indicando, como era esperado, que a difusão em relação ao próprio gradiente é mais importante do que a difusão devido à interação entre o soluto e o solvente (ZORRILLA; RUBIOLO, 1994; LOMBARDI; ZARITZKI, 1996). A simulação desprezando o número de Biot apresentou desvios de $5,76 \%$ e 2,95\%, respectivamente para a sacarose e água, valores próximos àqueles obtidos considerando a resistência externa. Portanto, o número de Biot estimado indica que a resistência externa pode ser desconsiderada no fenômeno de transferência estudado (SCHWARTZBERG; CHAO, 1982).

As Figuras 4 e 5 a seguir mostram, ao longo do tempo, o perfil de difusão da sacarose e da água, respectivamente, com os valores das concentrações obtidos experimentalmente e com os obtidos pormeio da aplicação do programa COMSOL Multiphysics 3.2 utilizando os valores dos coeficientes de difusão, principais e cruzados, da relação $\mathrm{h}_{\mathrm{m} /} \lambda_{\mathrm{m}}$ e do número de Biot otimizados no processo de desidratação em estudo (Tabela 4).

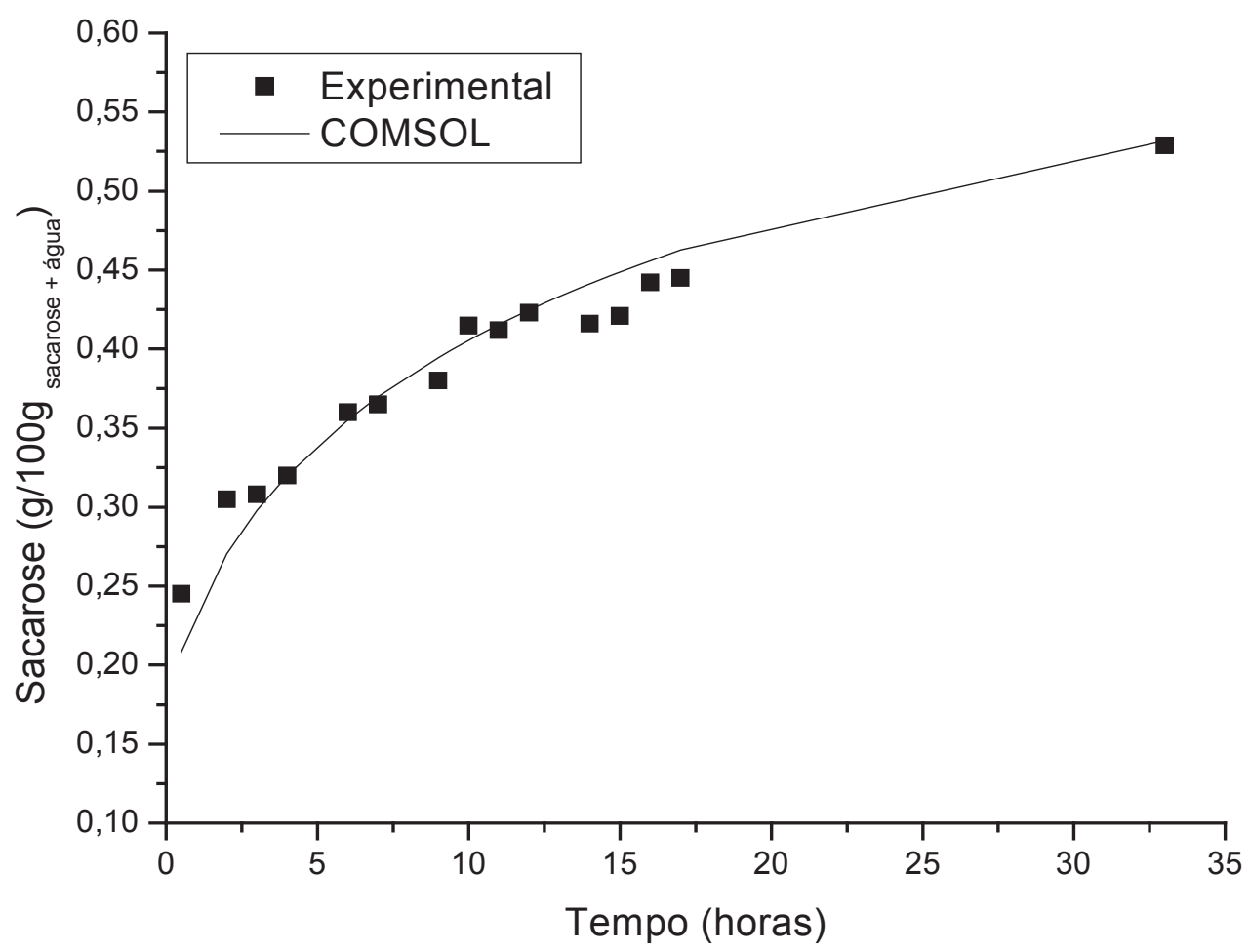

Figura 4. Perfil de distribuição da concentração de sacarose durante a desidratação osmótica de pedaços de maçã. Os pontos representam os dados experimentais e a linha a simulação. 


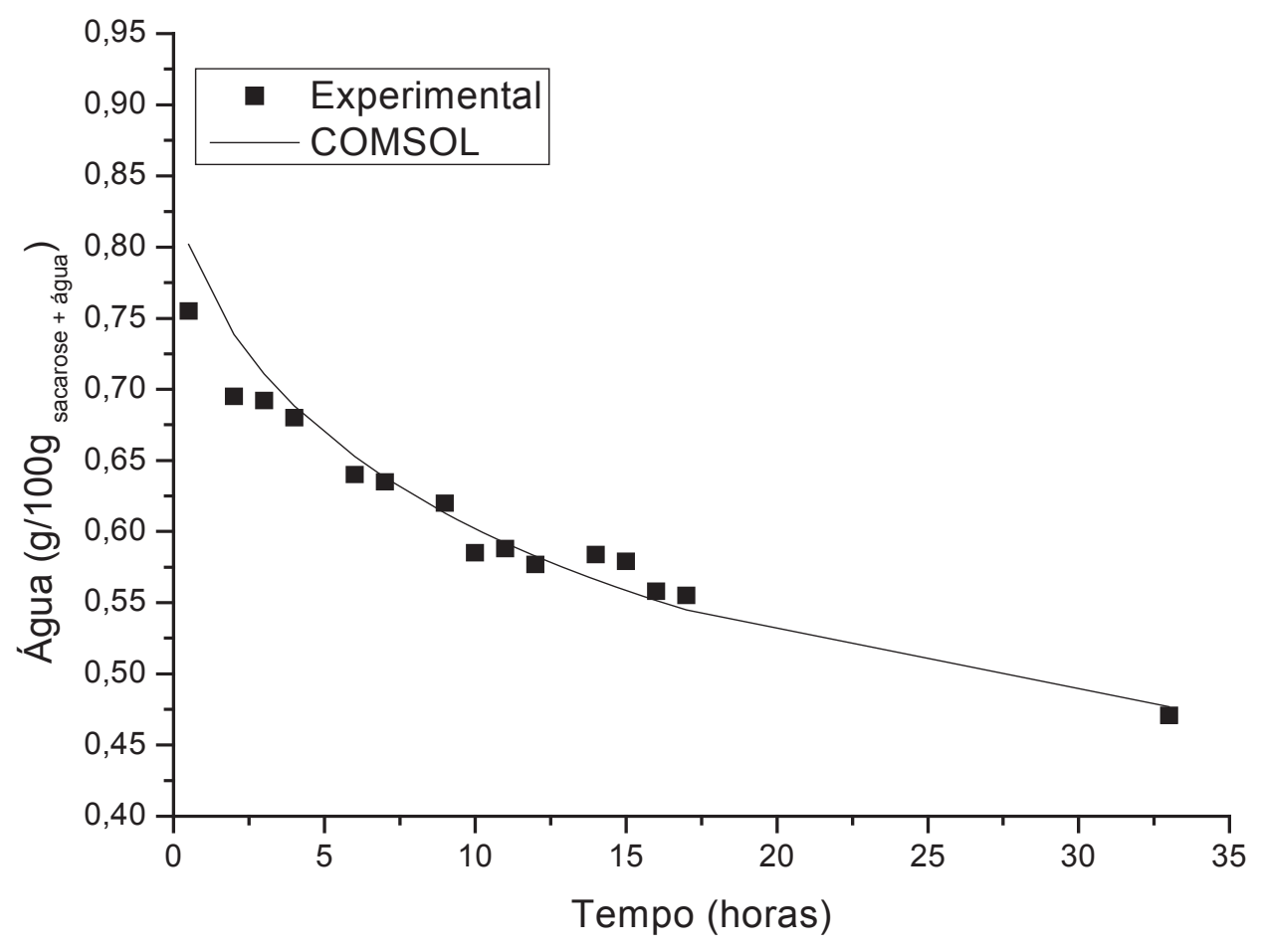

Figura 5. Perfil de distribuição da concentração de água durante a desidratação osmótica de pedaços de maçã. Os pontos representam os dados experimentais e a linha a simulação.

A análise dos resultados obtidos (Figura 5) para a desidratação osmótica de cilindros de maçã mostrou que a diminuição do teor de água, ao final do experimento, foi de $55 \%$ e ocorreu mais intensamente nas 12 primeiras horas do processo de desidratação, momento em que já se havia eliminado $34 \%$ do conteúdo inicial de água.

A Figura 6, que mostra o perfil de distribuição de sacarose (I) e água (II) após 33 horas de simulação do processo de desidratação osmótica, indica que ainda não houve a equalização da distribuição da sacarose bem como da água.
A Figura 6. I mostra um teor de 58,5\% de sacarose nas extremidades e $39,0 \%$ no centro do pedaço de maçã. A Figura 6. II mostra um teor de $41,5 \%$ de água nas extremidades e $60,1 \%$ no centro do cilindro de maçã indicando que após 33 horas de desidratação não temos, ainda, equalização das concentrações.

A Figura 7 mostra, em cortes, o perfil de distribuição da sacarose (I) e da água (II) após 90 horas de simulação onde a concentração prevista no centro do cilindro é de aproximadamente $97 \%$ do conteúdo de água e de sacarose na solução. 

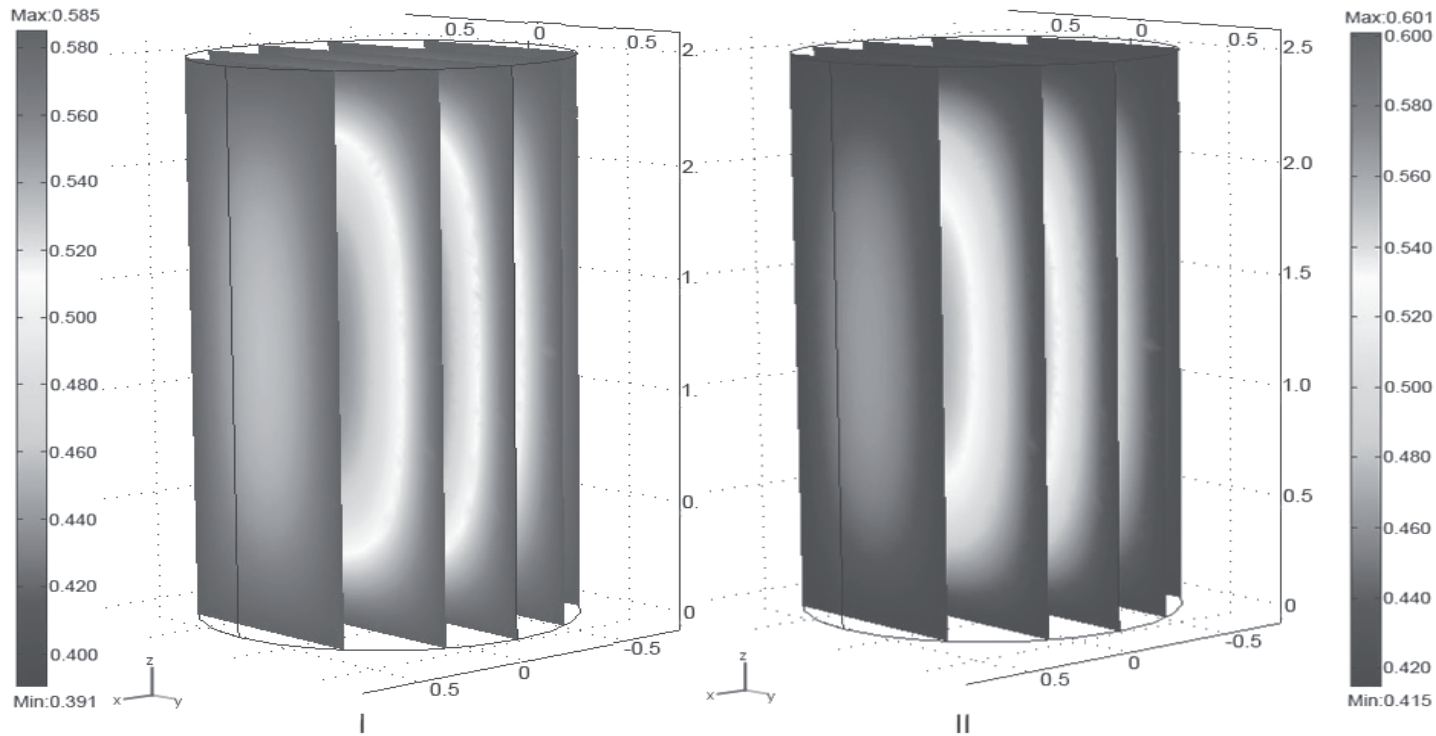

Figura 6. (I) Perfil de distribuição de sacarose e (II) perfil de distribuição de água após 33 horas de simulação da difusão multicomponente da desidratação osmótica.
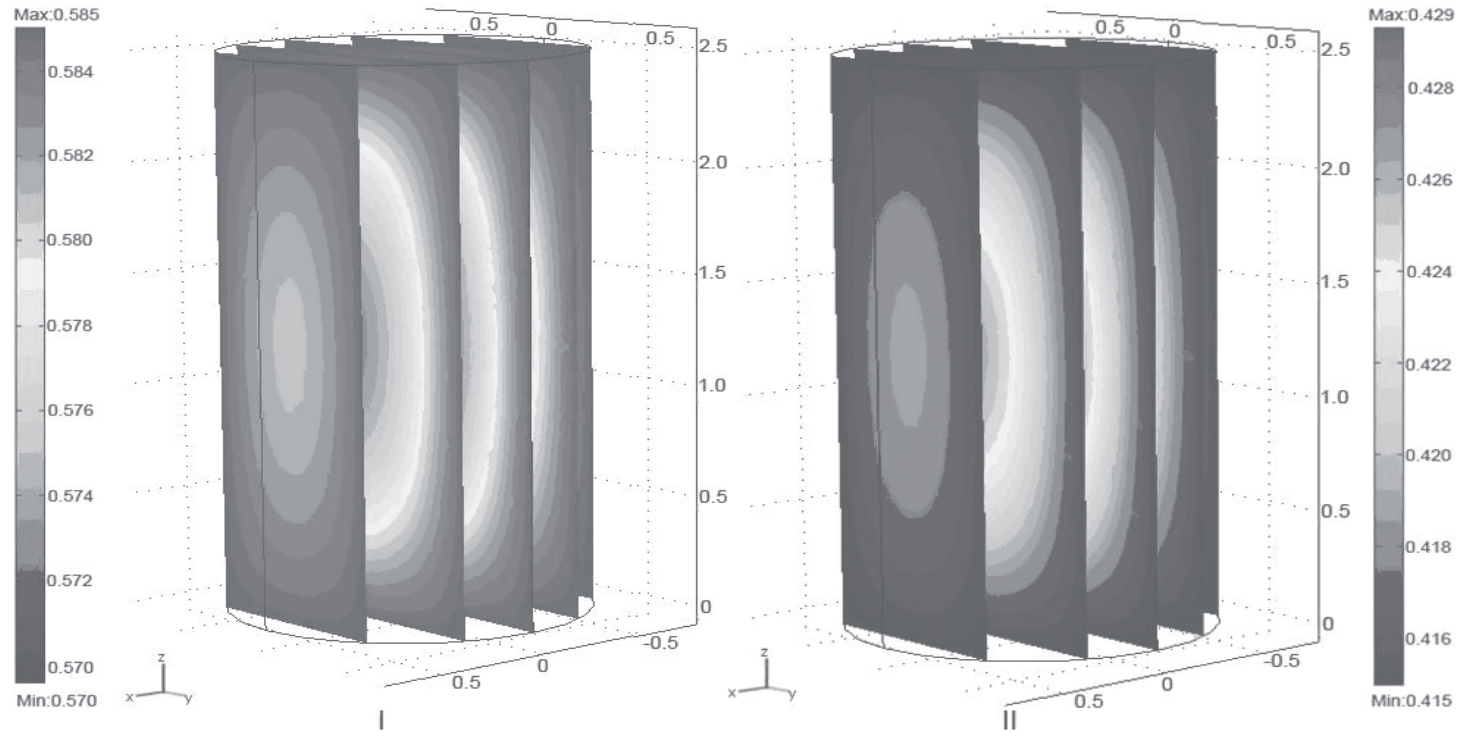

Figura 7. (I) Perfil de distribuição de sacarose e (II) perfil de distribuição de água após 90 horas de simulação. 


\section{Conclusões}

O método de otimização simplex acoplado às funções de desejabilidade mostrou ser uma ferramenta eficaz na busca dos principais parâmetros envolvidos no processo de difusão durante a desidratação osmótica da maçã.

Com a aplicação do método dos elementos finitos foi possível simular a desidratação osmótica de maçã. Os dados simulados para o processo de desidratação mostraram-se coerentes e convergentes com os resultados experimentais, validando a aplicação do MEF para a desidratação osmótica.

O MEF pode ser adaptado para o cálculo da concentração média do soluto e água na maçã, permitindo a estimativa da concentração de equilíbrio dos mesmos. Esta informação pode ter importância para a modulação do tempo de desidratação e, possível, melhoria na produtividade e maior controle da qualidade do processo.

\section{Agradecimentos}

Agradecimentos à Fundação Araucária pelo suporte financeiro e a UEL e CNPq pela concessão de bolsas.

\section{Referências}

AKTAS, T.; FUJII, S.; KAWANO, Y.; YAMAMOTO, S. Effect of some osmotic pre-drying treatments on drying kinetics, desorption isotherms and quality of vegetables. In: INTERNATIONAL DRYING SYMPOSIUM, 15., 2006, Gödöllö, Hungary. Proceedings... Gödöllö, Hungary: Szent István University Publisher, 2006. p. 877-883.

\section{AOAC - ASSOCIATION OF OFFICIAL} AGRICULTURAL CHEMISTS. Official methods of analysis of the association of analitical chemists. 14. ed. Washington, 1984, $1141 \mathrm{p}$.

BEVERIDGE, G. S. D.; SCHECHTER, R .S. Optimization: theory and practice. Tókio: McGraw-Hill Kogakusha, 1970. 773 p.
BONA, E.; BORSATO, D.; SILVA, R. S. S. F.; HERRERA, R. P. Aplicativo para otimização empregando o método simplex seqüencial. Acta Scientiarum, Maringá, v. 22, n. 5, p. 1201-1206, 2000.

BONA, E.; CARNEIRO, R. L.; BORSATO, D.; SILVA, R. S. S. F.; FIDELIS, D. A. S.; SILVA, L. H. M. E. Simulation of $\mathrm{NaCl}$ and $\mathrm{KCl}$ mass transfer during salting of prato cheese in brine with agitation: a numerical solution. Brazilian Journal of Chemical Engineering, Campinas, v. 24, n. 3, p. 337-349, 2007.

CARSLAW, H. S.; JAEGER, J. C. Conduction of heat in solids. 2. ed. London: Oxford University Press, 1959.

CASTRO, I. A.; SILVA, R. S. F.; TIRAPEGUI, J.; BORSATO, D.; BONA, E. Simultaneous optimization of response variables in protein mixture formulation: constrained simplex method approach. International Journal of Food Science and Technology, Oxford, v. 38, n. 2, p. 103-110, 2003.

CHANDRA, P. K.; SINGH, R. P. Applied numerical methods for food and agricultural engineers. Boca Raton: CRC Press Inc, 1995.

CHUNG,T. J. Finite element analysis in fluid dynamics. New York: McGraw-Hill, 1978, 378 p.

CRANK, J. The mathematics of diffusion. 2. ed. London: Oxford University Press, 1975.

DEROSSI, A.; PILLI, T. de.; SEVERINI, C.; MCCARTHY, M. J. Mass transfer during osmotic dehydration of apples. Journal of Food Engineering, Oxford, v. 86, n. 4, p. 519-528, 2008.

DERRINGER, G.; SUICH, R. Simultaneous optimization of several response variables. Journal of Quality Technology, Milwaukee, v. 12, n. 4, p. 214-219, 1980.

DIXON, G. M.; JEN, J. J. Changes of sugar and acid of osmotic dried apple slices. Journal of Food Science, Chicago, v. 42, n. 4, p. 1126-1131, 1977.

EIRAS, S.; ANDRADE, J. C. O uso do simplex modificado como estratégia de otimização em química analítica. Química Nova, São Paulo, v. 19, n. 4, p. 25-29, 1996.

FITO, P.; CHIRALT, A.; BETORET, N.; GRAS, M.; CHÁFER, M.; MARTÍNEZ-MONZÓ, J.; ANDRÉS, A.; VIDAL, D. Vacuum impregnation and osmotic dehydration in matrix engineering. Application in functional fresh food development. Journal of Food Engineering, Oxford, v. 49, n. 2, p. 175-183, 2001.

GHEZ, R. A primer of diffusion problems. New York: John Wiley \& Sons, 1988. 243 p. 
HAWKES, J.; FLINK, J. M. Osmotic concentration of fruit slices prior to freeze dehydration. Journal of Food Processing and Preservation, Mariland, v. 2, n. 4, p. 265284, 1978.

KHIN, M. M.; ZHOU, W.; YEO, S. Y. Mass transfer in the osmotic dehydration of coated apples cubes by using maltodextrin as the coating material and their textural properties. Journal of Food Engineering, Oxford, v. 81, n. 3, p. 514-522, 2007.

LAZARIDES, H.N.; KATSANIDES, E.; NICOILAIDES, A. Mass transfer kinetics during pre-concentration aiming at minimal solute uptake. Journal of Food Engineering, Oxford, v. 25, n. 2, p. 151-166, 1995.

LEWICKI, P. P.; LENART, A. Osmotic dehydration of fruits and vegetables. In: MUJUMDAR, A. S. (Ed.). Handbook of industrial drying. 3 rd ed. Boca Raton, FL: Taylor \& Francis, 2007. p. 665-687.

LOMAURO, G. L.; BAKSHI, A. S. Finite element analysis of moisture diffusion in stored foods. Journal of Food Science, Chicago, v. 50, n. 2, p. 392-396, 1985.

LOMBARDI, A. M.; ZARITZKY, N. E. Simultaneous diffusion of citric acid and ascorbic acid in prepeeled potatoes. Journal of Food Process Engineering, Malden, v. 19, n. 1, p. 27-48, 1996.

LUNA, J. A.; BRESSAN, J. A. Mass transfer during brining of cuartirolo argentino cheese. Journal of Food Science, Chicago, v. 51, n. 3, p. 829-831, 1986.

LYRA, P. R. M. Finite elements analysis of parabolic problems: combined influence of adaptive mesh refinement and automatic time step control. Revista Brasileira de Ciências Mecânicas, Rio de Janeiro, v. 15, n. 2, p. 172-198, 1993.

MANNHEIM, C. H.; LIU, J. X.; GILBERT, S. G. Control of water in foods during storage. Journal of Food Engineering, Oxford, v. 22, n. 3, p. 509-532, 1994.

NANJUNDASWAMY, A. M.; RADHAKRISHNAIAH SETTY, G.; BALACHANDRAN, C.; SAROJA, S.; MURTHY REDDY, K. B. S. Studies on development of new categories of dehydrated product from indigenous fruit. Indian Food Packer, New Delhi, v. 22, n. 1, p. 9193, 1978.
ONSAGER, L. Theories and problems of liquid diffusion. Ann. Sci., New York, v. 46, n. 5, p. 241-265, 1945.

PANADES, G.; CASTRO, D.; CHIRALT, A.; FITO, P.; NUÑEZ, M.; JIMENEZ, R. Mass transfer mechanisms occurring in osmotic dehydration of guava. Journal of Food Engineering, Oxford, v. 87, n. 3, p. 386-390, 2008.

PONTING, J. D. Osmotic dehydration of fruits - recent modifications and applications. Process Biochemistry, Watford, v. 8, n. 12, p. 18-20, 1973.

RAOULT-WACK, A. L. Recent advances in the osmotic dehydration of foods. Trends in Food Science \& Technology, Norwich, v. 5, n. 8, p. 255-260, 1994.

RICHARDSON, G. H. Standard methods for examination of dairy products. 15. ed. Washgington D. C.: American Public Health Association, 1985. 412 p.

SCHWARTZBERG, H. G.; CHAO, R. Y. Solute diffusivities in leaching processes. Food Technology, Chicago, v. 36, n. 2, p. 73-86, 1982.

TORREGGIANI, D. Osmotic dehydration in fruit and vegetable processing. Food Research International, Monticelo, v. 26, n. 1, p. 59-68, 1993.

WALTERS, F. H.; MORGAN, S. L.; PARKER, L. R.; DEMING, S. N. Sequential simplex optimization. Karskrona, Sweden: Licensed Eletronic Reprint by Multisimplex AB, 1999. 403 p.

YAO, Z.; LE MAGUER, M. Analysis of mass transfer in osmotic dehydration based on profiles of concentration, shrinkage, transmembrane flux and bulk flow velocity in the domain of time and space. J. Food Process Engineering, Malden, v. 20, n. 5, p. 401-414, 1997.

ZHANG, T.; BAKSHI, A. S.; GUSTAFSON, R. J.; LUND, D. B. Finite element analysis of nonlinear water diffusion during rice soaking. Journal of Food Science, Chicago, v. 49, n. 1, p. 246-250, 1984.

ZORRILLA, S. E.; RUBIOLO, A. C. A Model for using the diffusion cell in the determination of multicomponent diffusion coeficients in gels or foods. Chemical Engineering Science, Oxford, v. 49, n. 13, p. 2123-2128, 1994. 
